\title{
ANALYSIS OF AN ENERGY MINIMIZATION METHOD FOR LOCATING TRANSITION STATES ON POTENTUAL ENERGY HYPERSURFACES
}

\author{
Michael J. ROTHMAN and Lawrence L. LOHR Jr. \\ Department of Chemistry, University of Michigan, Ann Arbor, Muchugan 48109, USA
}

Received 10 Novermber 1979; in final form 14 December 1979

\begin{abstract}
Conditıons are given for the successful search for a transition state by an energy minimization method. Proofs for these gurdelines are presented. Advantages of this method are discussed, includung its use in establishing lower bounds to transitoon state energies Comparisons are made with other searching methods.
\end{abstract}

\section{Introduction}

The theoretical description of a chemical reaction often involves the search of a multi-dimensional potential energy hypersurface for a saddle point, or col, which might represent the "transition state" separating the "reactant" region of the hyperspace from the "product" region. The hypersurface itself is typically obtained from an approximate solution of the BornOppenheimer electronic Schrodinger equation. The properties of a hypersurface at a saddle point have been discussed by many authors [1-9]. Specifically the potentral energy second-derivative, or hessian, matrix possesses one and only one negative elgenvalue at a saddle point of the type that might correspond to a transition state. Throughout our discussion we shall use the term "saddle point" to refer exclusively to this type of extremum. The principal direction corresponding to the negative eigenvalue is the path of steepest descent away from the saddle pount and is often called the "reaction coordinate". The descripton of thus coordinate in terms of bond lengths and/or angles of the "supermolecule" compnsed of all atoms participating in the elementary reaction differs in general at the transition state from its description in either the asymptotic reactant or product regions. Thus if the location and nature of the saddle point is not known a priori, it may not be practical simply to follow the reaction coordinate "in" from the product region until a maximum is found in the potential ener- gy profile. The development of systematic procedures to locate saddle points does not appear to have received the attention which has been devoted to procedures to locate maxuma or minima. Indeed optimization procedures, such as steepest ascent (descent) methods [10], are often selected so as to avoid convergence to saddle points. However several methods utilizing the norm of the gradient have been developed and applied by McIver and Komornicki $[6,11-13]$ to the location of saddle pounts. They have done this within the context of semi-empirical molecular orbital methods, partıcularly MINDO/2, in which gradients are easily computed. Varıous ab initio gradient procedures have been developed [14-18], including a recent one [19] for use with multiconfiguration self-consistent-field (MC SCF) methods. We now outline a "traditional" energy minimization method for locating saddle points and analyze its salient characteristics. In the second part of this study we present [20] its application to a MC SCF study of the $\mathrm{H}_{2}+\mathrm{D}_{2}$ exchange reaction.

\section{General method}

We wish to have a systematic procedure to locate a saddle point with one and only one negative eigenvalue of the hessian matrix when the reaction coordinate is unknown. Our procedure is smply stated:

(1) Given the potential energy $V(x)$ as a function of $3 N-6=n$ internal degrees of freedom $x=\left\{x_{1}\right.$, 
$\left.x_{2}, \ldots, x_{n}\right\}$ for the supermolecule, select one coord1nate, say $x_{n}$, as a reference, or "distınguished", coordinate.

(2) Minimize $V(x)$ for fixed $x_{n}$. This requires an optimization in an $(n-1)$-dimensional subspace and yields a function $\mathcal{O}\left(x_{n}\right)$

(3) Vary $x_{n}$ until $\mathcal{V}\left(x_{n}\right)$ is a maximum The associated pathway is an $(n-1)$-component vector $\boldsymbol{P}\left(\boldsymbol{x}_{n}\right)$.

McCullough and Silver [21] have compared a similar energy minimization path for a two-dimensional surface to the path of steepest descent. Their definition of the path differs from ours in that they employ different choices of $x_{n}$ on different sides of the saddle point. However, they simply assumed that their minimization path passes through the saddle point, which as we shall show need only be the case for continuous $P\left(x_{n}\right)$ from reactants to products. Since the energy minimization method is very useful in some instances, we wish to explore its features in some details

We now cxamune the conditions for which the maxımum of $\mathcal{V}\left(x_{n}\right)$ corresponds to the desired saddle point of $V(x)$. First we note that a maximum of $\mathcal{V}\left(x_{n}\right)$ is necessanly an extremum of $V(x)$; this follows from the fact that a function whose gradient is zero in $n-1$ directions and in an arbitrary direction having a non-zero component normal to the $\boldsymbol{n}-1 \mathrm{~d}$ rections, must have a zero gradient in the $n$th orthogonal direction.

Let the $\boldsymbol{n}$ internal coordinates be represented by $\boldsymbol{x}$ $\equiv\left\{x_{1}, x_{2}, \ldots, x_{n}\right\}$. In the neighborhood of an extremum,

$V(x)=\frac{1}{2} \sum_{i=1}^{n} a_{l} x_{l}^{2}+\sum_{i<j}^{n} b_{i j} x_{l} x_{l}+K$,

where $K$ is a constant and the origin $\theta \equiv\{0,0, \ldots, 0\}$ is at the extremum. Principal directions $\hat{u} \equiv\left\{u_{1}\right.$, $\left.u_{2}, \ldots, u_{n}\right\}$ may be chosen such that

$V(\bar{u})=\frac{1}{2} \sum_{l=1}^{n} c_{l} u_{l}^{2}+K$.

If the extremum is a saddle point corresponding to a transition state, then one and only one $c_{l}$ is negative, say $c_{n} \cdot$

$c_{\imath}>0, \quad \imath=1,2, \ldots, n-1$,

$c_{l}<0, \quad i=n$.
The direction $u_{n}$ is the reaction coordinate at the saddle point. Defining durections $\hat{v} \equiv\left\{v_{1}, v_{2}, \ldots, v_{n}\right\}$, with $v_{n}=x_{n}$, such that cross-terms vanish within the $n-1$ space $\left\{v_{1}, v_{2}, \ldots, v_{n-1}\right\}$,

$$
V(\hat{v})=\frac{1}{2} \sum_{i=1}^{n} d_{i} v_{i}^{2}+\sum_{i=1}^{n-1} e_{i n} v_{i} v_{n}+K \text {. }
$$

In the appendix we employ (4) to demonstrate that a maximum in $\mathcal{V}\left(x_{n}\right)$ can occur only at a saddle point of $V(x)$, provided that the pathway $P\left(x_{n}\right)$ is continuous along some segment containung the maximum. However, the stronger condition that the pathway lead continuously from reactants to products must be satisfied in order to guarantee the occurrence of a maxsmum in $\mathcal{V}\left(x_{n}\right)$ at a saddle point in the neighborhood of which the quadratic form (1) obtains.

To illustrate the preceding consider

$$
V(x, y)=-a(x-y)^{2}+b(x+y)^{2}
$$

for points $(x, y)$ in the neighborhood of the saddle point at $(0,0)$. The path of steepest descent and its energy profile are given by $x=-y$ and $\mathcal{V}_{\text {sd }}(y)$ $=-4 a y^{2}$, respectively. The profile given by the minimization criterion is $\mathcal{V}(y)=-4 a b y^{2} /(b-a)$ for the case $b>a$, so that $\mathcal{V}(y) \leqslant \mathcal{V}_{\text {sd }}(y)$, with the equallty holding for $y=0$. The path being followed in the minimization procedure is $x(y)=-(b+a) y /(b-a)$, so that $\boldsymbol{x}(0)=0$, meaning that this path intersects the path of steepest descent at the saddle point. However, if $b<a$, attempted minimization with respect to $x$ for fixed $y$ yields $V(x, y) \rightarrow-\infty$ as $x \rightarrow \pm \infty$, so that the search leads away from the saddle point into a region for which the quadratic approximation of (5) is unadequate. On the general surface approxımated by (5) two minima will exist for $y=0$, both lower in energy than the transition state. The pathway $x_{r}(y)$ starting from "reactants" will differ from the pathway $x_{\mathrm{p}}(y)$ starting from "products". At some $y, V\left(x_{\mathrm{r}}, y\right)=V\left(x_{\mathrm{p}}, y\right)$ and there will be a discontinuity in the pathway $x(y)$ defined as the locus of global (as opposed to local) minima.

Before continuing we note an important inequality. Let $\mathcal{V}_{\text {sd }}\left(x_{n}\right)$ denote values of the potential energy at points $s$ along the path of steepest descent from the saddle point, with these points being "addressed" by their projections onto $x_{n}$. Since $\mathcal{V}\left(x_{n}\right)$ is defined in our method to be a mumum with respect to varia- 
tion of the $n-1$ coordinates $x_{1}, x_{2}, \ldots, x_{n-1}$ for fixed $x_{n}$, it follows that

$\mathcal{V}\left(x_{n}\right) \leqslant \mathcal{V}_{\text {sd }}\left(x_{n}\right) \leqslant V_{\text {ts }}$,

where $V_{\mathrm{ts}}$ is the transition state energy.

The first equality holds for $x_{n}=0$ under the conditrons previously stated, in which case both $\mathcal{W}\left(x_{n}\right)$ and $\mathcal{V}_{\text {sd }}\left(x_{n}\right)$ have maxima at $x_{n}=0$. Note from (6) that $\mathcal{V}\left(x_{n}\right)$ is a lower bound to $V_{t s}$ for all $x_{n}$.

Thus we have shown that the profile $\mathcal{V}\left(x_{n}\right)$ will under some but not all curcumstances include the transition state energy. If the pathway $P\left(x_{n}\right)$ leads continuously from reactants to products, then from (6) and the definition of the transition state at the lowest barrier between reactants and products there is necessarily a maximum in $\mathcal{V}\left(x_{n}\right)$ at the transition state geometry and with the transition state energy. Thus, a maximum in the profile $\mathcal{V}\left(x_{n}\right)$ is a necessary condition for a "transition state" saddle point under this condition. However, a maximum in the profile $\mathcal{V}\left(x_{n}\right)$ is also a sufficient condition for the desired saddle point, since there is one and only one negative eigenvalue of the $n$-dimensional hessian matrix of (4) if the $d_{1}$ are $>0$ for $i=1,2, \ldots, n-1$, a result (see appendix) which is independent of $d_{n}$ and the $n-1$ coupling coefficients $e_{i n}$ in (4) Thus if a maximum in $\mathcal{V}\left(x_{n}\right)$ is located and $P\left(x_{n}\right)$ is continuous in that region, no further testing is required to ascertain that a saddle point has been found.

\section{Choice of distinguished coordinate}

A successful search for a saddle point depends cntically upon the choice of the "distinguished" coordinate $x_{n}$ which, as a first condition, must have a non-zero projection onto the path of steepest descent at the desired saddle point. Clearly some choices of $x_{n}$ lead to discontinuous pathways, such occurrences being the major shortcoming of the minumization method. We noted that a discontinuity occurs, so that the saddle point is not located, when there does not exist a minimum (for finite values of the coordinates) in the $(n-1)$-dimensional subspace for a quadratically approximated potential energy surface. A negative eigenvalue of the $(n-1)$-dimensional hessian matrix for the subspace may anse if the mixed second derivatives $V_{1 n} \equiv \partial^{2} V / \partial x_{1} \partial x_{n}$ for $i=1,2, \ldots, n-1$ are suf- ficiently large in magnitude. Within the quadratic approximation of (4), the derivatives $V_{2 n}$ are simply the coefficients $e_{i n}$. From (4) the components of the pathway $P\left(x_{n}\right)$ are $P_{1}\left(x_{n}\right)=-\left(e_{i n} / d_{i}\right) x_{n}$, so that as $e_{i n}$ $\rightarrow 0, P_{z}\left(x_{n}\right) \rightarrow 0$ and the pathway coincides with the path of steepest descent. Thus it is desirable to choose $x_{n}$ so as to minimize the sum of the squares of the $V_{i n}$ for points in the neighborhood of the desired saddle point.

\section{Comparisons with other methods}

In the method of Komornicki et al. [16] several points are calculated along a linearly interpolated pathway between reactants and products. Starting from the geometry corresponding to the maximum energy along this pathway a search for the transition state is made using analytic gradient methods with a variant of a gencralized Newton-Raphson technique to minimize $\sigma$, which is the sum of squares of first derivatives of the potential energy. Since $\sigma$ minimization may serve to locate any extremum, a second derivative test must be employed to ascertain that a given extremum is a saddle point with one and only one negative eigenvalue. As we have shown (see appendix) no such test is required with the energy minimization method.

Halgren and Lipscomb [22] have developed the "synchronous transit" method, which also employs a linearly interpolated pathway in the first stage of the search. A minumization is then carried out subject to the constraint of fixed coordinate on the linear synchronous transit (LST) path. A quadratic synchronous transit (QST) path is then constructed which passes through this minimum as well as through reactant and product geometries. A coincidence of a maximum on the QST pathway (an upper bound to the transition state energy) with the orthogonally optimized mir:mum (a lower bound) indicates the successful location of a transiton state. We note that if the LST pathway coordinate is identified with our "distinguished" coordinate $x_{n}$, then the orthogonally optimized structure corresponds to our $(n-1)$-dimensional minimum for the particular value of $x_{n}$ corresponding to the LST maximum. The chief advantage of the methods of Halgren and Lipscomb and of Komornicki et al. is the economy resulting from the reduced dimensionality of the initial stage of the search. 
We note finally that the initial search along a linear synchronous pathway, which is common to both the methods of Komornickı et al, and of Halgren and Lupscomb, could also be used in connection with the energy minimization method to narrow the search. The three methods then differ in their approaches to the final $n$-dimensional search for the transition state.

\section{Summary}

An analysis has been presented of an energy minimization method for locating "transition state" saddle points on potentral energy hypersurfaces. This "trad1tional" reaction coordinate method does not require an a priori knowledge of the nature of the reaction pathway. A maxunum in the potential energy along a minimization pathway is proven to occur necessarily at a saddle point provided that the pathway leads continuously from reactants to products and that the surface is describable by a quadratic form in the neighborhood of the saddle point Under the weaker condition that the pathway on such a surface be continuous only along some segment including a maximum, it is further proven that a maximum can only uccur at a saddle point. All points along the pathway have energies which are lower bounds for the transition state energy.

\section{Appendix}

As we noted in the text, a maxımum along $\mathcal{V}\left(x_{n}\right)$ corresponds to an extremum in $V(x)$ This, in most cases, justifies the representation of the surface in this neighborhood with (4). Specifically the use of (4) is valid if $P\left(x_{n}\right)$ is continuous along a segment of $x_{n}$ containing the maximum. Here we demonstrate that a maximum in $\mathcal{V}\left(x_{n}\right)$ is the desired saddle point of $V(x)$

It can be shown given the $n$-dumensional hessian matrix $A$ of (4), that

$\operatorname{det} A=\left[\prod_{i=1}^{n} d_{i}\right]-\sum_{i=1}^{n-1}\left\{e_{2 n}^{2}\left[\prod_{\substack{j=1 \\ j \neq 1}}^{n-1} d_{j}\right]\right\}$.

At each point along the minimization path $\boldsymbol{P}\left(x_{n}\right)$, $\partial V(u) / \partial v_{1}=0, \quad i=1,2, \ldots, n-1$.

Utilizing these $n-1$ constraints with (4) we obtain

$\mathcal{V}\left(x_{n}\right)=\frac{1}{2}\left[d_{n}-\sum_{i=1}^{n-1} e_{i n}^{2} / d_{i}\right] x_{n}^{2}+K$.

At the maximum of $\mathcal{V}\left(x_{n}\right)$ along $P\left(x_{n}\right)$,

$\mathrm{d}^{2} \mathcal{V}\left(x_{n}\right) / \mathrm{d} x_{n}^{2}<0$,

so that from (A.3)

$d_{n}<\sum_{i=1}^{n-1} e_{l n}^{2} / d_{l}$

Substitution of (A.5) into (A.1) yrelds the upper bound $\operatorname{det} \mathbf{A}<0$,

since minimization implies that $d_{1}>0$ for $i=1,2$, $\ldots, n-1$. Thus det $A$ is negative, indicating an odd number of negative eigenvalues of $A$. At this point we introduce a separation theorem [23] for eigenvalues.

Theorem. The elgenvalues $\lambda_{1}^{\prime}, \ldots, \lambda_{n-1}^{\prime}$ of the matrix obtained by omitting the last row and column of a hermitean matrx $M$ separate the eigenvalues $\lambda_{1}, \ldots$, $\lambda_{n}$ of $\mathrm{M}$

A being real and symmetric implies $A$ is hermitean. Omitting the $n$th row and column of $A$ yields an $(n-1)$-dimensional matrix with all positive eigenvalues $d_{l}, i=1,2, \ldots, n-1$. Since the eigenvalues of $A$ are separated by the eigenvalues of the $(n-1)$ dimensional matrix, there is exactly one eigenvalue of $A, \lambda$, such that $\lambda<d_{1}, i=1,2, \ldots, n-1$. (The eigenvalues are assumed here to be distinct, i.e., non-degenerate.) Above we concluded that there were an odd number of negative eigenvalues of $A$, now we see that there is only one eigenvalue of $A$ that may be negative. Therefore, there is one and only one negative eigenvalue of the hessian matrix $A$ at the maximum of $\mathcal{V}\left(x_{n}\right)$. This is the requirement for a saddle point to serve as a "transition state".

\section{References}

[1] J.N. Murrell and K.J. Laidler, Trans. Faraday Soc 64 (1968) 371 . 
[2] J.N. Murrell and G.L. Pratt, Trans Faraday Soc. 66 (1970) 1680.

[3] D.M. Bishop and K.J. Laidler, Trans. Faraday Soc. 66 (1970) 1685.

[4] K. Fukui, J. Phys. Chem. 74 (1970) 4161.

[5] J.W. McIver Jr. and R.S. Stanton, J. Am. Chem Soc. 94 (1972) 8618.

[6] J.W. McIver Jr and A. Komornick1, J. Am. Chem. Soc. 94 (1972) 2625.

[7] R.E. Stanton and J.W. McIver Jr., J. Am Chem. Soc. 97 (1975) 3632

[8] E. Pollak and P. Pechukas, J. Am. Chem. Soc. 100 (1978) 2984.

[9] D.R. Coulson, J. Am. Chem. Soc. 100 (1978) 2992.

[10] D J. Wilde, Optumum seeking methods (Prentice-Hall, Englewood Cuffs, 1964) pp. 107-121.

[11] J.W. McIver Jr. and A. Komornick1, Chem. Phys. Letters $10(1971) 303$.

[12] A. Komornicki and J.W. Mclver Jr, J. Am. Chem. Soc. 95 (1973) $4512 ; 96$ (1974) 5798; 98 (1976) 4553.

[13] M C. Flanigan, A. Komornicki and J.W McIver, in. Modern theoretical chemistry, Vol 8, ed G.A. Segal (Plenum Press, New York, 1977) pp 1-47.
[14] D. Poppinger, Chem. Phys. Letters 34 (1975) 332; 35 (1975) 550.

[15] H.B. Schlegel, S. Wolfe and F. Bernardi, J. Chem. Phys. 63 (1975) 3632 .

[16] A. Komornicki, K. Ishida, K. Morokuma, R. Ditchifield and M. Conrad, Chem. Phys. Letters 45 (1977) 595.

[17] M. Dupuis and H.F. King, J. Chem. Phys. 68 (1978) 3998

[18] J.A Pople, R. Krishnan, H.B. Schlegel and J.S. Binkley, Intern. J. Quantum Chem. 14 (1978) 545.

[19] J.D. Goddard, N.C. Handy and H.F. Schaefer III, J. Chem. Phys. 71 (1979) 1525.

[20] M.J. Rothman, L.L. Lohr Jr., C.S. Ewig and J.R. van Wazer, to be published.

[21] E A. McCullough Jr. and D.M. Silver, J. Chem. Phys. 62 (1975) 4050.

[22] T A. Halgren and W.N. Lipscomb, Chem. Phys. Letters 49 (1977) 225.

[23] B. Noble, Applied lnear algebra (Prentice-Hall, Englewood Cliffs, 1969) pp. $415,416$. 